

\section{(III) UNIVATES}

Universidade do Vale do Taquari - Univates

Reitor: Prof. Me. Ney José Lazzari

Vice-Reitor e Presidente da Fuvates: Prof. Dr. Carlos Cândido da Silva Cyrne

Pró-Reitora de Pesquisa, Extensão e Pós-Graduação: Profa. Dra. Maria Madalena Dullius

Pró-Reitora de Ensino: Profa. Dra. Fernanda Storck Pinheiro

Pró-Reitora de Desenvolvimento Institucional: Profa. Dra. Júlia Elisabete Barden

Pró-Reitor Administrativo: Prof. Me. Oto Roberto Moerschbaecher

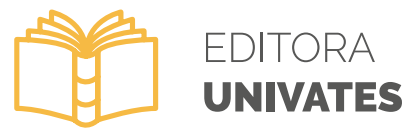

\section{Editora Univates}

Coordenação: Ana Paula Lisboa Monteiro

Editoração e capa: Glauber Röhrig e Marlon Alceu Cristófoli

\section{Conselho Editorial da Editora Univates}

\section{Titulares}

Alexandre André Feil

André Anjos da Silva

Fernanda Rocha da Trindade

João Miguel Back

Sônia Elisa Marchi Gonzatti

\section{Suplentes}

Fernanda Cristina Wiebusch Sindelar

Claudete Rempel

Adriane Pozzobon

Rogério José Schuck

Evandro Franzen

Avelino Tallini, 171 - Bairro Universitário - Lajeado - RS, Brasil

Fone: (51) 3714-7024 / Fone: (51) 3714-7000, R.: 5984

editora@univates.br / http://www.univates.br/editora

S578 Signos / Fundação Alto Taquari de Ensino Superior. - Ano 1 (1975) - Lajeado, RS: Ed. da Univates, 2019.

Ano 40, n. 1, 2019.

Instituição mudou o nome para Unidade Integrada Vale do Taquari de Ensino Superior em 1997.

Instituição mudou o nome para Centro Universitário Univates em 1999.

Editora mudou o nome para Ed. da Univates em 1999.

Instituição mudou o nome para Universidade do Vale do Taquari Univates em 2017.

Semestral.

ISSN 1983-0378

1. Linguística. 2. Linguagem. 3. Literatura. 4. Leitura. 5. Comunicação - Ensino. I. Universidade do Vale do Taquari - Univates.

CDU: 8

Catalogação na publicação - Biblioteca da Univates

Bibliotecária Andrieli Mara Lanferdini - CRB 10/2279

\section{Os artigos são de exclusiva responsabilidade do(s) autor(es).}

(c) Fundação Vale do Taquari de Educação e Desenvolvimento Social - FUVATES 


\section{SLELTOS}

SIGNOS é uma publicação semestral com foco no Ensino. É mantida pela UNIVATES, estando aberta à submissão de artigos nacionais e internacionais. A primeira edição, Ano 1, foi publicada em 1975.

\section{Conselho Executivo}

Prof. Dr. Rogério José Schuck (Editor Geral)

\section{Conselho Editorial}

Prof. Dr. Alfredo Rocha de la Torre, Universidad Pedagógica Y Tecnológica De

Colombia, Colômbia

Profa. Dra. Ana Maria Tramunt Ibaños, PUCRS, Brasil

Prof. Dr. Ângelo Vitório Cenci, UPF, Brasil

Prof. Dr. Cezar Luís Seibt, UFPA, Brasil

Profa. Dra. Cleci Teresinha Werner da Rosa, UPF-ICEG, Brasil

Prof. Dr. Evandson Paiva Ferreira, UFG-CEPAE, Brasil

Prof. Dr. Fábio Steyer, UEPG, Brasil

Profa. Dra. Gilcilene Dias da Costa, UFPA, Brasil

Prof. Dr. Javier Marins Ceballos, Universidad de Murcia, Espanha

Prof. Dr. Lúcio Paulo do Amaral Crivano Machado, UERJ-IBRAG, Brasil

Profa. Dra. Mairim Linck Piva, FURG, Brasil

Profa. Dra. Maria Cristina Ferreira dos Santos, UERJ, Brasil

Prof. Dr. Miguel Ángel Villamil Pineda, Universidad Santo Tomás, Colômbia

Prof. Dr. Miguel Rettenmaier da Silva, UPF, Brasil

Prof. Dr. Phd Ricardo Manuel das Neves Vieira, Instituto Politécnico de Leiria, Portugal

Profa. Dra. Rosangela Gabriel, UNISC, Brasil

Profa. Dra. Sani de Carvalho Rutz da Silva, UTFPR, Câmpus Ponta Grossa, Brasil 


\section{AGRADECIMENTOS}

A Comissão de Editores da Signos agradece a todos os pareceristas que colaboram com a qualidade dos artigos da revista, através da análise e sugestões para melhoria dos manuscritos submetidos à publicação.

Altair Fávero (UPF)

Andreia Aparecida Guimarães Strohschoen (UNIVATES)

Angelica Vier Munhoz (UNIVATES)

Cezar Luís Seibt (UFPA)

Cristiane Antonia Hauschild (UNIVATES)

Danise Vivian (UNIVATES)

Dante Diniz Bessa (UNISINOS)

Derli Juliano Neuenfeldt (UNIVATES)

Fabiane Olegário (UNIVATES)

Flávio Roberto Meurer (UNIVATES)

Ieda Maria Giongo (UNIVATES)

Italo Gabriel Neide (UNIVATES)

Itamar Luís Hammes (IFSUL)

Jacqueline Silva da Silva (UNIVATES)

João Miguel Back (UNIVATES)

José Claudio Del Pino (UNIVATES)

Kári Lúcia Forneck (UNIVATES)

Lauro Inacio Ely (UNIVATES)

Lúcio Jorge Hammes (UNIPAMPA)
Marcelo Leandro dos Santos (UNIVATES)

Márcia Jussara Hepp Rehfeldt

(UNIVATES)

Márcia Solange Volkmer (UNIVATES)

Maria Elisabete Bersch (UNIVATES)

Maria Madalena Dullius (UNIVATES)

Maristela Maristela Juchum (UNIVATES)

Marli Teresinha Quartieri (UNIVATES)

Mateus Dalmaz (UNIVATES)

Miriam Miriam Ines Marchi (UNIVATES)

Morgana Domênica Hattge (UNIVATES)

Nélia Maria Pontes Amado (UALG - PT)

Rosmari Terezinha Cazarotto (UNIVATES)

Sandro Frohlich (UNIVATES)

Silvana Rossetti Faleiro (UNIVATES)

Sonia Elisa Marchi Gonzatti (UNIVATES)

Suzana Suzana Feldens Schwertner

(UNIVATES)

Vilmar Alves Pereira(FURG) 


\section{APRESENTAÇÃO}

A presente publicação da Revista Signos mantém o foco no Ensino e está organizada em três grandes blocos de discussão. No primeiro bloco, a atenção se volta para a supervisão educacional em contextos e sistemas educativos latino-americanos. O primeiro artigo destaca a importância da supervisão nos processos administrativos e pedagógicos, culminando com a análise em torno de transformações nos sistemas educativos na América Latina. O segundo e terceiro artigos adentram na questão da subjetividade, especialmente nos discursos que fabricam subjetividades docentes no ensino e na educação feminina. O quarto e último artigo deste bloco trata de uma formação específica, a saber, o ensino superior militar. Percebeu-se que os instrutores militares têm uma boa formação específica na sua área, mas carecem de preparação em termos pedagógicos para o exercício da docência.

O segundo bloco inicia com o quinto artigo, que trata dos novos ambientes de aprendizagem e do debilitamento das fronteiras da escola, sendo estes ambientes construídos por sujeitos que organizam territórios delimitados física e historicamente. Já o sexto artigo adentra no fenômeno das altas habilidades, investigando uma escola pública no interior do Mato Grosso do Sul/MS. Chama atenção para a necessidade de práticas docentes que potencializam os indivíduos em termos de desenvolvimento da criatividade. Fechando este bloco, que gira em torno das fronteiras do ensino, o sétimo artigo aborda a necessidade de trabalharmos com recursos didáticos multimodais, diante de um contexto pós-moderno que traz as tecnologias no ensino, aplicando-os ao ensino de Geografia e História.

O terceiro e último bloco de discussões traz, no oitavo texto, a questão do conhecimento significativo e, no caso do nono texto, questões referentes à cognição. A seguir, o décimo texto apresenta uma reflexão acerca das motivações de leitura junto ao jovem brasileiro, bem como uma crítica ao senso comum de que "o jovem brasileiro não gosta de ler". Fechando a presente edição, o décimo primeiro artigo faz uma análise a respeito da aquisição de linguagem escrita de crianças em processo de alfabetização, aproximando-se à percepção de que a escrita se processa junto a um sujeito imerso na linguagem. Na certeza de estarmos colocando à disposição do leitor um excelente material que partilha o saber científico, damos por concluída mais uma Edição da Revista Signos.

Prof. Dr. Rogério José Schuck

Editor Geral da Revista Signos 


\section{SUMÁRIO}

\section{LA SUPERVISIÓN EDUCATIVA EN EL CONTEXTO DE LOS}

SISTEMAS EDUCATIVOS LATINOAMERICANOS 8

Ernesto Fajardo Pascagaza

RETRATO FALADO DO PROFESSOR: DISCURSOS QUE FABRICAM SUBJETIVIDADES DOCENTES. 26

Maria das Graças Moura Santos

Dhemersson Warly Santos Costa

Carlos Augusto Silva e Silva

\section{A EDUCAÇÃO ESCOLAR POR NÍSIA FLORESTA EM UMA} ANÁLISE DO DISCURSO FRANCESA

Erika Caroline de Oliveira Cavalcanti

Nadia Pereira da Silva Gonçalves de Azevedo

ENSINAR E APRENDER DE INSTRUTORES E DE ALUNOS MILITARES 55

Deise Becker Kirsch

Maria da Graça Nicoletti Mizukami

EL DISEÑO DE NUEVOS AMBIENTES DE APRENDIZAJE Y EL DEBILITAMIENTO DE LAS FRONTERAS DE LA ESCUELA 75

Miller Antonio Pérez Lasprilla

\section{ALTAS HABILIDADES/SUPERDOTAÇÃO: A CRIATIVIDADE} COMO UM DE SEUS TRAÇOS DETERMINANTES 98

Mariana Patricia Soares de Oliveira

Bárbara Amaral Martins 
OS RECURSOS DIDÁTICOS MULTIMODAIS E AS TECNOLOGIAS NO ENSINO DE GEOGRAFIA E HISTÓRIA NA PÓS-

MODERNIDADE 121

Augusto Russini

Elsbeth Léia Spode Becker

TRADUÇÃO COMO CONSTRUÇÃO DE CONHECIMENTO: EXPERIÊNCIAS NA UNIVERSIDADE DE BRASÍLIA 136

Ana Helena Rossi

NOTAS SOBRE O PENSAMENTO DE BENTO PRADO JR.: AS CIÊNCIAS COGNITIVAS E A IPSEIDADE 150

Eduardo Toshio Kobori

Rita Ferreira da Silva

Fábio Ságula de Oliveira

“O JOVEM BRASILEIRO NÃO GOSTA DE LER (O QUÊ? E POR QUÊ??)": REFLETINDO SOBRE A QUESTÃO A PARTIR DE DADOS DA PESQUISA RETRATOS DA LEITURA 158

Paulo Ailton Ferreira da Rosa Junior

ENTRE A PSICOGÊNESE E A LINGUÍSTICA: UMA ANÁLISE

EM AQUISIÇÃO DE LINGUAGEM ESCRITA DE CRIANÇAS EM PROCESSO DE ALFABETIZAÇÃO .174

Maria Aparecida Ramos Lima

Dennys Dikson 